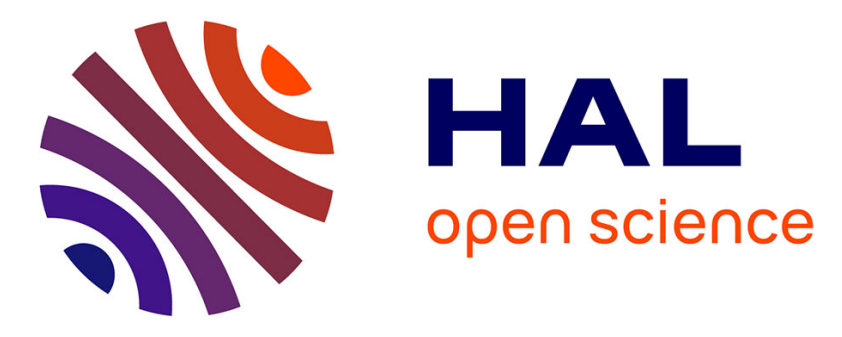

\title{
Cognition et performance collectives en sport
}

Carole Sève, Jérôme Bourbousson, Germain Poizat, Jacques Saury

\section{To cite this version:}

Carole Sève, Jérôme Bourbousson, Germain Poizat, Jacques Saury. Cognition et performance collectives en sport. Intellectica - La revue de l'Association pour la Recherche sur les sciences de la Cognition (ARCo), 2009, Sport de haute performance et cognition, 52, pp.71-95. 10.3406/intel.2009.1199 . hal00570468

\section{HAL Id: hal-00570468 https://u-bourgogne.hal.science/hal-00570468}

Submitted on 7 Jun 2021

HAL is a multi-disciplinary open access archive for the deposit and dissemination of scientific research documents, whether they are published or not. The documents may come from teaching and research institutions in France or abroad, or from public or private research centers.
L'archive ouverte pluridisciplinaire HAL, est destinée au dépôt et à la diffusion de documents scientifiques de niveau recherche, publiés ou non, émanant des établissements d'enseignement et de recherche français ou étrangers, des laboratoires publics ou privés. 


\title{
Cognition et performance collectives en sport
}

\author{
Carole SEVE ${ }^{1}$, Jérôme BOURBOUSSON ${ }^{1}$, \\ Germain POIZAT ${ }^{2}$, Jacques SAURY
}

RESUME : Cet article présente, dans un premier temps, les éléments clefs d'un courant nommé «Team Cognition» (ou cognition collective), initialement développé dans le domaine de la psychologie dite «Industrielle / Organisationnelle », et auquel un certain nombre de chercheurs sur le sport se réfèrent actuellement. Pour les auteurs de ce courant, le fonctionnement et les performances des équipes doivent être étudiées en accordant une place centrale aux phénomènes cognitifs permettant aux différents membres d'une équipe de se coordonner. Ces phénomènes sont conçus en termes de partage, appréhendé en tant que produit et processus, de « contenus cognitifs » entre les partenaires. Dans un deuxième temps, sont présentés les résultats majeurs d'études menées avec différentes équipes sportives (tennis de table en double, voile en équipage, basket-ball). Ces études se sont principalement attachées à la caractérisation du partage de la situation d' « ici et maintenant». Les résultats concernent le caractère incertain et fluctuant du partage d'informations contextuelles, les processus de co-construction d'une intelligibilité mutuelle sur la base du partage de ces informations, et les modes de coordination au sein de l'équipe en résultant. Ils offrent de nouveaux éléments de compréhension concernant la cognition et la performance collectives d'équipes sportives mais aussi plus largement de toute équipe visant l'accomplissement d'une performance collective: équipe de travail, équipe d'intervention en milieu hostile (militaires, pompiers, sauveteurs), ensemble de création artistique, etc.

Mots clés : Cognition collective, activité collective, sport, intelligibilité mutuelle, coordination.

ABSTRACT: Team Cognition and Team Performance in Sport. First, this paper presents the key elements of a research program labeled "Team Cognition", originally developed in the field of industrial/organizational psychology. Leaders of this field of research estimate that team functioning and team performance have to be studied with regard to the cognitive phenomena involved in team coordination. These phenomena are considered in terms of cognitive contents sharing (sharing as a product and sharing as a process) between teammates. This line of research brings new perspectives on team expertise in sport. Recently some studies in sport focused on the content and process of sharing between partners. Thus, we secondly present the main results of recent studies on team cognition in different sports (table tennis doubles, sailing crews, basketball teams). These studies mainly principally investigated the shared context (i.e., the nature and content of the contextual information that was shared between the partners). Results underlined the dynamic and indeterminated properties of contextual information sharing, the co-construction process of mutual intelligibility, and

* 1 Université de Nantes ; 2 Université de Bourgogne

Carole Sève : UFRSTAPS, 25 Bd Guy Mollet, BP 72206. 44322 Nantes Cedex 3

Carole.seve@univ-nantes.fr

(C) 2009 Association pour la Recherche Cognitive. 
coordination modes between team's members. These results lead to new elements of understanding about collective performance in sport, and more generally about collective activity in work situations.

Key words: Team Cognition, collective activity, sport, mutual intelligibility coordination.

\section{LE COURANT DE LA « TEAM COGNITION »}

Depuis quelques années se développe une ligne de recherche identifiée sous le vocable de "Team Cognition », s'attachant au fonctionnement des équipes (e.g., Cooke \& Gorman, 2006; Eccles \& Johnson, 2009; Eccles \& Tenenbaum, 2004, 2007 ; Fiore \& Salas, 2006). Un de ses objectifs essentiels est de comprendre pourquoi une «équipe experte » ne peut se réduire à une " équipe d'experts », et en quoi la performance collective diffère de la somme des performances individuelles des membres de l'équipe. Les recherches conduites sous cette dénomination générale se sont initialement développées dans les domaines de la psychologie dite "Industrielle / Organisationnelle » (I/O psychology) (Cooke et al., 2007; Salas \& Fiore, 2004), avec pour principales sources d'inspiration les théories cognitivistes du traitement de l'information (e.g., Hinsz et al., 1997), et celles de la cognition socialement partagée et distribuée (e.g., Hutchins, 1995 ; Resnick et al., 1991). Pour les auteurs de ce courant, le fonctionnement et les performances des équipes doivent être étudiés en accordant une place centrale aux phénomènes cognitifs permettant aux différents membres d'une équipe de se coordonner. Ces phénomènes sont conçus, d'une façon générale, en termes de partage de « contenus cognitifs » entre les partenaires. Ces contenus sont identifiés, selon les auteurs, à l'aide de différentes notions : « connaissances partagées » (shared knowledge) (Eccles \& Tenenbaum, 2004 ; Navarro, 1991), « modèles mentaux partagés » (shared mental models) (Cannon-Bowers et al., 1993), référentiel commun (de Terssac \& Chabaud, 1990 ; Hoc, 2001, 2003 ; Loiselet \& Hoc, 2001), contexte partagé (Salembier \& Zouinar, 2004). Ce partage est appréhendé en tant que produit (ce qui est partagé en termes de contenus) et processus (la manière dont se crée le partage, autrement dit les modes de coordination des activités individuelles).

\section{I.1. Le contenu du partage}

Les études s'attachent à la caractérisation de deux types de contenu cognitifs (a) des contenus cognitifs relativement statiques et préexistants à l'activité collective, et (b) des contenus cognitifs plus transitoires et dynamiques.

\section{I.1.1. Les contenus cognitifs pré-existant à l'activité collective}

Ces contenus renvoient aux connaissances construites par les membres de l'équipe au cours de leurs expériences et interactions passées. Ces connaissances sont organisées sous forme de modèles mentaux. La notion de modèles mentaux partagés (MMP) (Cannon-Bowers et al., 1993) rend compte chez les membres de l'équipe d'une même organisation de connaissances similaires. Des études menées dans divers domaines (aviation civile et militaire, centre de régulation) ont montré le rôle essentiel de ces modèles 
mentaux pour la réalisation d'une performance collective (Cannon-Bowers et al., 1993 ; Mathieu et al., 2000 ; Salas et al., 1992 ; Stout et al., 1999).

La littérature sur les modèles mentaux partagés distingue classiquement deux types de MMP jouant un rôle dans la régulation des coordinations d'actions entre les membres d'une équipe :

- les modèles mentaux relatifs à la tâche à accomplir (« taskwork mental models »). Leur contenu décrit et organise les connaissances sur la manière d'accomplir la tâche collective. Il se réfère, entre autres, aux procédures collectives «standard» à accomplir, aux stratégies génériques à mettre en œuvre, aux conditions environnementales et technologiques, aux critères de réussite de la tâche, etc.

- les modèles mentaux relatifs à l'organisation du travail d'équipe (« teamwork mental models »). Leur contenu décrit et organise les connaissances quant au fonctionnement d'une équipe en particulier. Il se réfère, entre autres, aux modalités spécifiques d'interactions entre les membres de l'équipe; aux statuts, responsabilités et rôles des différents membres de l'équipe; aux habiletés, préférences, forces, faiblesses, habitudes des partenaires, etc.

La distinction entre ces deux types de MMP explique, par exemple, que bien que tous les footballeurs d'un même niveau d'expertise puissent partager un ensemble de connaissances génériques relatives aux techniques de jeu et aux stratégies d'organisation (e.g., une " défense individuelle », une " défense en ligne »), ils ne sont pas pour autant capables d'être d'emblée efficaces lors de leur intégration dans une nouvelle équipe, car celle-ci a développé dans le cours de son histoire collective, des formes spécifiques d'organisation, des codes de communication propres ou des tactiques de jeu singulières, que le joueur arrivant doit apprendre.

Deux aspects des modèles mentaux apparaissent comme des prédicteurs essentiels de la performance collective: le degré de similitude entre les modèles mentaux des membres de l'équipe (similarité du contenu des connaissances et des liens entre les connaissances, c'est-à-dire la structure), et la justesse de ces modèles (pertinence et exactitude du contenu des connaissances). La similitude et la justesse des modèles mentaux partagés sont identifiées à l'aide de différentes techniques: entretiens, questionnaires, méthodes dites " conceptuelles » (représentation des concepts d'un domaine et de leurs relations). A titre d'illustration, une étude de Lim et Klein (2006) a examiné la relation entre la similarité et la justesse des modèles mentaux et la performance collective en situation naturelle chez 71 équipes de militaires (4 à 8 membres). Dans un premier temps, les chercheurs ont évalué la similitude et la justesse des modèles mentaux des différents membres de l'équipe. L'évaluation des modèles mentaux relatifs à la tâche et au fonctionnement de l'équipe de chaque sujet a été réalisée de la manière suivante: chaque participant devait juger du lien de parenté entre 14 concepts correspondant à la tâche et 14 concepts correspondant à l'équipe, en utilisant une échelle de 1 (complètement indépendant) à 7 (fortement relié). La similarité des modèles mentaux des membres d'une même équipe a été évaluée à l'aide du calcul de la proportion de liens communs entre les membres de l'équipe sur la totalité des liens existants. L'indice de justesse des modèles mentaux de chaque participant 
a été obtenu sur la base de la comparaison de la moyenne de ces modèles mentaux avec ceux d'experts du domaine. Le score de justesse de chaque équipe a été calculé en moyennant les indices de justesse des modèles mentaux des membres de cette équipe. Dans un second temps, les équipes ont participé à des tests de combat lors desquels la performance collective a été évaluée par des officiers supérieurs. Les résultats montrent que les équipes dans lesquelles les membres présentent des contenus de connaissances structurés et organisés de la même manière réalisent de meilleures performances, et confirment l'idée que la similarité et la justesse des modèles mentaux relatifs à la tâche et au fonctionnement de l'équipe sont des prédicteurs de la performance collective.

\section{I.1.2. Les contenus cognitifs transitoires et dynamiques}

Les contenus cognitifs transitoires et dynamiques nécessaires au fonctionnement harmonieux de l'équipe ont, quant à eux, essentiellement été appréhendés en lien avec le concept de " conscience de la situation » (situation awareness) (Endsley, 1995; Sarter \& Woods, 1991). Cette conscience est envisagée de manières différentes selon les options théoriques et méthodologiques des recherches. Dans certains cas, l'accent est porté sur la perception immédiate de la situation, dans d'autres le concept de conscience de la situation englobe, de manière plus globale, différents processus cognitifs. Dans le domaine de la psychologie ergonomique, la conscience de la situation est souvent définie comme " la perception d'éléments de l'environnement à l'intérieur d'un volume spatio-temporel, la compréhension de leur signification et la projection de leur statut dans un futur proche » (Endsley, 1995). Elle représente ainsi l'état cognitif dynamique et transitoire de l'acteur, c'est-à-dire la perception et la compréhension qu'il a de son environnement à l'instant $t$. Elle intègre des projections dans le futur, réalisées sur la base de la mise en relation de jugements portés sur la situation actuelle et de connaissances récupérées en mémoire à long terme et permet la construction d'un modèle de la situation. La conscience de la situation et le modèle de la situation qui en résulte sont dynamiques, en ce sens qu'ils évoluent en permanence, en relation avec la perception de nouveaux éléments dans la situation qui modifient les attentes de l'acteur quant à son devenir.

L'éventail de méthodes pour évaluer la conscience de la situation est très large. Salmon et al. (2006) dénombrent plus de 30 techniques utilisées au cours des 20 dernières années. De manière générale, les chercheurs accèdent à la conscience de la situation d'un acteur par le biais de verbalisations simultanées (des acteurs en train de réaliser une tâche sont invités à décrire les éléments pris en compte, les connaissances mobilisées au cours de l'activité), de verbalisations interruptives lors «d'arrêt sur image » d'une simulation (après un arrêt de la simulation à un point critique du scénario simulé, les acteurs sont questionnés sur leur conscience de la situation), ou de verbalisations $a$ posteriori (rappel stimulé après la réalisation d'une tâche). Les études sur la conscience de la situation ont essentiellement été menées dans les domaines de l'aviation civile et militaire, de la conduite automobile, des centres de contrôle divers (appels d'urgence, trafic routier, aérien ou ferroviaire). De manière synthétique les résultats montrent que les experts font preuve, par comparaison avec les novices, d'une plus grande capacité à regrouper les informations présentes dans la situation, d'une meilleure habileté à donner du sens aux 
informations perçues, et d'une capacité plus importante à se projeter dans le futur et construire des attentes quant à l'évolution de la situation. Aussi avec la pratique, les acteurs, en développant leur conscience de la situation et leur capacité à ajuster leur modèle de la situation, deviennent plus aptes à exploiter les opportunités offertes par le décours d'une situation.

Cependant si cette notion de conscience de la situation rend compte de l'aspect dynamique et transitoire des contenus cognitifs d'un individu et de la construction d'un modèle de la situation par celui-ci, elle ne rend pas compte des contenus cognitifs transitoires partagés entre différents membres. Pour pallier ce manque, différents auteurs (e.g., Cooke et al., 2001; Stout et al., 1996) ont proposé le concept de "Team Situation Awareness », que nous traduisons par « conscience collective de la situation». Tout en se fondant sur la conscience de la situation de chacun des membres de l'équipe, cette conscience collective de la situation ne se réduit pas à la somme de ces «consciences individuelles». Salas et al. (1995) pointent notamment l'émergence de «processus comportementaux collectifs» (« team process behaviors ») sans pour autant apporter beaucoup de précisions sur ces derniers. Stout et al. (1996) ont proposé une modélisation pour rendre compte des relations entre processus comportementaux collectifs et conscience de la situation à un niveau individuel et collectif.

Cette modélisation précise le concept de conscience collective de la situation et le modèle collectif de la situation qui en résulte, en présentant les relations entre différents contenus cognitifs et les contraintes environnementales au niveau individuel et collectif. Cependant elle n'est associée à aucune méthodologie précise pour évaluer cette conscience collective et ce modèle collectif. Aussi les méthodologies visant à appréhender cette forme de partage restent à construire. A notre connaissance, seuls Gorman, Cooke et Winner (2006) ont, jusqu'alors, proposé une méthode pour rendre compte de la conscience collective de la situation. Cette méthode, nommée "CAST », (Coordinated Awareness of Situation by Teams), permet de décrire et de mesurer la coordination des perceptions et des actions émergeant des interactions dynamiques entre les membres d'une équipe, à partir d'un codage de leurs communications au cours de la résolution d'un problème auquel ils sont confrontés dans des conditions expérimentales.

\section{I.1.3. Formes de partage et formats d'équipe}

Le partage de contenus cognitifs entre les membres d'une équipe sportive apparaît ainsi comme le garant d'un fonctionnement collectif efficace, en ce sens qu'il permet la construction d'interprétations congruentes et d'attentes communes. Ces attentes communes, en permettant d'anticiper les actions des autres membres de l'équipe, facilitent la coordination des activités individuelles. Le partage des contenus cognitifs relatifs à une tâche collective peut être envisagé de différentes manières. Cooke et al. (2007) proposent trois formes caractéristiques de partage (Figure 1).

- similarité : les membres de l'équipe ont des contenus cognitifs, relatifs à la tâche collective, isomorphes ; 
- complémentarité : les contenus cognitifs des membres de l'équipe, tout en présentant une part commune, sont différents ;

- distribution : les contenus cognitifs sont différents les uns des autres.
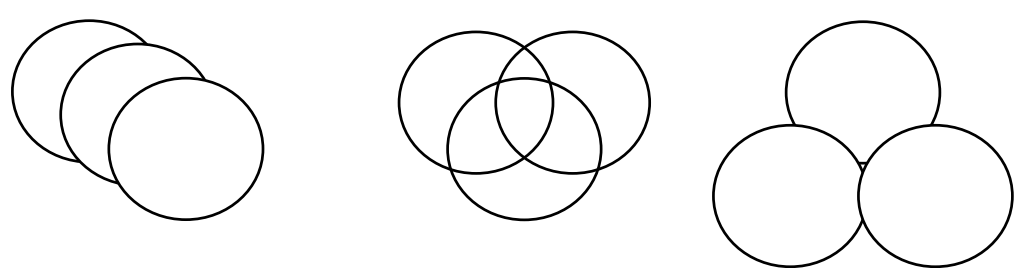

Degré de spécialisation des rôles

Figure 1 : Formes de partage des connaissances selon Cooke et al. (2007)

Selon Cooke et al. (2007), la forme du partage requis pour un fonctionnement collectif efficient dépend des exigences de la tâche collective. Plus les exigences de coordination entre les membres de l'équipe sont fortes et plus le degré de spécialisation des sous-tâches individuelles est faible, et plus la similarité des contenus cognitifs est cruciale. Les équipes fonctionnant sur ce modèle sont dites «homogènes" (Cooke \& Gorman, 2006; Cooke et al., 2007). Par contre, pour des équipes dans lesquelles les exigences de coordination sont moindres et le degré de spécialisation des rôles plus marqué, le partage s'effectue davantage sur le mode de la distribution: chacun des individus possède un domaine de compréhension qui lui est propre, et leur l'agencement avec ceux des autres membres permet à l'équipe d'être efficace. Les équipes de ce type sont dites " hétérogènes » et présentent généralement une répartition des rôles de chaque individu fortement organisée en amont de la performance collective.

\section{I.2. Les processus de partage}

Un deuxième axe essentiel de recherche du courant Team Cognition concerne la manière dont s'effectue et se régule le partage des contenus cognitifs entre les membres d'une équipe, dans des situations dynamiques et incertaines. Ces processus de partage ont essentiellement été appréhendés en termes d'échanges d'informations et de communications. La dynamique du partage d'informations et des communications dans le cours de l'activité collective constitue une manifestation des processus de coordination cognitive au sein des équipes. Ces échanges et communications peuvent être verbaux ou non verbaux, intentionnels ou non intentionnels, explicites ou implicites. Les possibilités de communication, et ainsi les processus de partage, diffèrent selon les contraintes pesant sur l'activité collective. Stout et al. (1996) distinguent trois contextes typiques de réalisation d'une performance collective dans des situations dynamiques et incertaines, et font des hypothèses concernant les 
processus de partage les plus pertinents au regard des caractéristiques de ces contextes.

Le premier contexte renvoie à des situations dans lesquelles les membres de l'équipe ont de nombreuses occasions d'échanger verbalement (avant et/ou en cours de la réalisation de la tâche collective). Dans ce cas, la coordination entre les membres se fonde sur des plans d'actions élaborés avant la performance collective et réajustés au cours de la performance. Les plans préparatoires permettent la répartition des rôles et responsabilités de chacun au sein de l'équipe et la détermination de séquences d'actions collectives. Ils sont réajustés en cours d'action sur la base de communications verbales explicites entre les membres. Ces réajustements s'opèrent lorsque des événements non prévus par le plan surviennent, les séquences d'actions envisagées inefficaces, les procédures et routines collectives habituelles mises à défaut. Ils constituent des opportunités pour "réviser » et affiner les bases de connaissances et les modèles mentaux partagés.

Le deuxième contexte renvoie à des situations dynamiques et incertaines dans lesquelles les possibilités d'échanges d'information entre les membres de l'équipe sont extrêmement réduites. Dans ce cas, un processus tacite de coordination (Wittenbaum et al., 1996) est nécessaire. Cette coordination est possible grâce à la construction d'attentes similaires quant au devenir de la situation, du fait de l'existence de modèles mentaux partagés préalables. Ces derniers permettent l'intégration des actions de chacun dans un décours temporel, grâce à la prévision des actions des partenaires qui permet d'agir au bon moment. Ces modèles mentaux partagés sont renforcés (ou non) selon le résultat de la coordination. Pour illustrer le type de partage requis pour réaliser une performance collective efficace dans ces situations, Stout et ses collaborateurs donnent l'exemple de la "passe aveugle» au basket-ball. Réussir une telle tâche (réaliser une passe sans regarder son partenaire) requiert que le porteur de balle sache où et quand le coéquipier sera disponible et que les partenaires eux-mêmes anticipent la passe et sachent où elle peut arriver. La réalisation efficace d'une telle passe nécessite ainsi des inférences de chaque joueur sur les actions à venir des coéquipiers, qui peuvent se réaliser grâce à l'extraction des mêmes indices sur l'évolution de la situation. Les joueurs, sur la base de ces indices, portent le même jugement sur la situation, mobilisent les mêmes modèles mentaux qui s'actualisent dans la réalisation d'un enchaînement d'actions individuelles pré-déterminées et coordonnées.

Le dernier contexte renvoie à des situations au cours desquelles les membres de l'équipe "informent» les autres de leurs intentions, grâce à des communications implicites, notamment des comportements moteurs ou verbaux porteurs de sens pour les coéquipiers. La signification de ces comportements résulte de la construction de codes communs, ou d'expériences communes antérieures qui se sont cristallisées dans des modèles mentaux partagés. Stout et ses collaborateurs illustrent cette forme de coordination en s'appuyant à nouveau sur l'exemple de la passe aveugle en basket-ball. Le porteur de balle peut, par des comportements moteurs particuliers, avertir ses partenaires de son intention de réaliser une passe aveugle. La perception de ces comportements oriente les attentes des coéquipiers. 
La distinction de ces trois contextes est réalisée en référence à un élément jugé essentiel pour l'échange d'informations: «les opportunités de coordination » (Cannon-Bowers \& Bowers, 2006). Ces opportunités sont liées à diverses contraintes, telles que la pression temporelle pesant sur les activités individuelles, le caractère discret ou continu des séquences d'actions individuelles et collectives, le positionnement spatial des acteurs et leur visibilité, le partage ou non de diverses ressources matérielles, ou encore les modalités de communication possibles. Ces échanges d'informations, et ainsi les opportunités de coordination, peuvent se réaliser à trois moments essentiels : (a) avant la réalisation de la performance collective (définition des buts, planification des stratégies, distribution des rôles et responsabilités), (b) dans le cours de la réalisation de la performance collective (communication intentionnelle et/ou non intentionnelle, explicite et/ou implicite, replanification), et (c) après la réalisation de la performance collective (débriefing, évaluation de l'efficacité des plans envisagés et des routines collectives) (Eccles \& Tenenbaum, 2004). Les contenus des informations échangées lors de ces trois moments ne sont pas de même nature et influent de manière différente sur les processus de régulation du partage et l'ajustement des modèles mentaux partagés.

\section{I.3. Paradigmes d'étude}

Les recherches menées dans le courant de la Team Cognition font apparaître des perspectives théoriques et méthodologiques diverses quand à l'étude du partage entre les membres d'une équipe sportive. Deux perspectives contrastées peuvent être identifiées.

La première correspond à ce que Cooke et al. (2007) nomment "perspective du traitement de l'information», ou encore "perspective agrégative » (Cooke \& Gorman, 2006). Selon cette perspective, la " team cognition " consiste en une addition, ou agrégation, de processus liés à la cognition individuelle des membres de l'équipe. Les études sont focalisées sur le partage - en termes de similitude ou de complémentarité - des connaissances (ou modèles mentaux), ou des "consciences de la situation" individuelles, et non sur les processus de partage. Cette perspective, considérée comme «individualiste» et «statique» par ces auteurs, est associée à des méthodes d'investigation par questionnaires, essentiellement basées (a) sur des comparaisons de similitude entre les contenus cognitifs des différents membres, et (b) sur la caractérisation des rôles spécifiques des membres de l'équipe et des interactions entre ces rôles. Si ces études apportent des connaissances quant au contenu du partage sous-jacent à la réalisation d'une performance collective, elles sont peu informatives quand aux processus de partage et ignorent le caractère incertain et dynamique de ce partage.

La deuxième perspective correspond à ce que Cooke et al. (2007) nomment la perspective "holistique, écologique et dynamique», qu'ils associent à l'acronyme «THEDA» ("Team Holistic Ecology and Dynamic Activity»). Elle regroupe différents courants de recherche (e.g., cognition socialement distribuée, psychologie écologique, théorie des systèmes dynamique, théorie de l'activité), et approche l'équipe en tant qu'entité cognitive dotée de propriétés globales, non réductibles à l'agrégation des processus cognitifs individuels. 
Elle se focalise, en conséquence, sur les processus et comportements collectifs caractérisant le fonctionnement de l'équipe, plutôt que sur les activités de ses membres, en utilisant des méthodes susceptibles d'évaluer directement la coordination des activités au sein de l'équipe, comme par exemple, la méthode «CAST » (Coordinated Awareness of Situation by Teams), ou encore, l'étude de la dynamique et des patterns de communication au sein des équipes (Cooke et al., 2007). Deux courants majeurs sont particulièrement représentatifs de cette perspective THEDA :

- la cognition distribuée (e.g., Hutchins, 1995). Ce courant envisage la cognition comme une propriété globale émergeant de processus distribués au sein du collectif. Dans cette perspective, la « conscience collective de la situation» d'une équipe émerge des processus d'interaction au sein de cette équipe (et entre cette équipe et son environnement), sans qu'aucun de ses membres ne possède en propre une représentation et une compréhension de la totalité de la situation. Les propriétés cognitives globales de l'équipe sont réparties dans les nœuds du système (intégrant les acteurs et leur environnement), sans être localisables dans un centre de traitement ou dans la «tête » de chaque composant (Hazlehurst et al. 2007). En cohérence avec ces présupposés, ce courant appréhende la performance collective et la cognition à travers le flux d'informations circulant entre les acteurs et leur environnement socio-technique ;

- l'analyse des communications entre les membres d'une équipe (e.g., Cooke et al., 2007). Ce courant accorde une place essentielle aux communications. Les communications verbales, dans certains contextes de travail en équipe, constituent les principales interactions entre les membres. Ces communications offrent un moyen d'accès privilégié et une « fenêtre ouverte » sur la « team cognition » (Pedersen \& Cooke, 2006). Pour Cooke et al. (2007), de la même manière que les protocoles de «pensée à voix haute » sont utilisés pour accéder aux processus cognitifs individuels (Ericsson \& Simon, 1980), les communications entre les membres d'une équipe de travail constituent une voie d'accès à la cognition collective. Dans cette optique, les propriétés cognitives de l'équipe sont appréhendées à travers l'analyse du flux, de la structure et du contenu des communications entre les membres de l'équipe au cours de la réalisation d'une performance collective.

Les recherches menées dans le courant de la Team Cognition ont été, pour leur majorité, réalisées dans les domaines de l'aviation (civile et militaire) et de la régulation du trafic (aérien, ferroviaire, et routier). Actuellement les perspectives théoriques et méthodologiques ouvertes par ces études, bien qu'elles soient stimulantes, ne constituent encore que des sources d'inspiration relativement spéculatives pour l'étude de l'activité des équipes sportives. Certains articles du numéro thématique de la revue International Journal of Sport and Exercise Psychology (e.g., Ward \& Eccles, 2006), consacré au courant de la Team Cognition, exposent les intérêts et limites, pour l'étude des performances sportives collectives, des perspectives théoriques et 
méthodologiques de ce courant et en appellent au développement d'études empiriques dans le domaine du sport.

\section{L'ACTIVITE COLLECTIVE EN SPORT}

\section{II.1. Des études menées en référence au « cours d'action »}

Récemment des travaux empiriques relatifs aux phénomènes de partage et à la coordination dans des équipes sportives (e.g., Bourbousson et al., 2008 ; Poizat et al., 2007, 2008 ; 2009 ; Saury, 2008b), ont été menés dans le cadre du programme du cours d'action (Theureau, 1992, 2006). Cette approche de l'activité collective est qualifiée de «situationnisme méthodologique" par Theureau (2006). Il la présente comme une voie moyenne entre «l'individualisme méthodologique » et le «collectivisme méthodologique » auxquels les deux perspectives précédemment présentées correspondent respectivement. Le terme de voie moyenne fait référence au fait que le cadre théorique et méthodologique d'analyse du cours d'action vise à appréhender, dans le même mouvement, les activités individuelles et l'activité collective. Ce cadre d'analyse propose plusieurs objets théoriques pour accéder à la dynamique des connaissances mobilisées et des significations construites par les acteurs en situation (Theureau, 2006). L'objet théorique «cours d'action » est une réduction de l'activité à sa partie qui est significative pour l'acteur : le cours d'action est «l'activité d'un acteur engagé dans une situation, qui est significative pour ce dernier, c'est-à-dire montrable, racontable et commentable par lui à tout instant, moyennant des conditions favorables » (Theureau, 2004, p.48). Il est constitué d'un enchaînement d'unités d'activité significatives émergeant de l'interaction de l'acteur avec sa situation. Ces unités articulent différentes composantes dont les attentes de l'acteur, les connaissances mobilisées, les éléments pris en compte dans la situation. Restituer le cours d'action d'un acteur consiste à identifier l'enchaînement de ces unités de façon à préciser les processus de construction de significations en action. L'activité collective, pour sa part, est appréhendée en considérant (a) qu'elle est autonome, c'est-à-dire que les interactions entre acteurs font émerger un domaine de phénomènes propre à la dynamique de l'activité collective, et (b) se construit sur la base de l'articulation des activités individuelles-sociales. L'analyse de l'objet théorique «articulation des cours d'action » de plusieurs acteurs vise à caractériser la manière dont l'activité collective se construit sur la base de la concaténation des activités individuelles.

Les études sur la performance collective en sport menées en référence au cadre théorique du cours d'action (e.g., Bourbousson et al., 2008 ; Poizat et al., 2007, 2008, 2009 ; Saury, 2008b) ont appréhendé le partage, sur la base de l'articulation des cours d'action des membres d'une même équipe sportive, en termes de ce qui était partagé ici et maintenant dans la situation (i.e., le partage de la situation actuelle significative pour les acteurs). Il s'agissait d'identifier le partage concernant les éléments pris en compte dans la situation présente, les attentes quand au devenir de la situation, les interprétations construites par les différents membres de l'équipe. Ce niveau d'appréhension du partage renvoie, 
dans la modélisation du courant de la Team Cognition, à la notion de situation awareness (i.e., la compréhension immédiate de la situation).

Etudier le partage de la situation actuelle significative pour les acteurs permet de dépasser le caractère relativement statique des modélisations du partage de connaissances stables et pré-existantes à l'activité collective et d'accéder à la dynamique même de cette activité collective. Ceci ouvre vers la compréhension de la co-construction d'une intelligibilité mutuelle entre les membres de l'équipe (Decortis \& Pavard, 1994 ; Salembier \& Zouinar, 2004), c'est-à-dire la possibilité de comprendre les intentions et attentes des autres protagonistes, et les processus de coordination entre les partenaires.

\section{II.2. Partage d'informations contextuelles et performance collective au sein de dyades sportives}

\section{II.2.1. La notion de contexte partagé}

Salembier et Zouinar (2004) ont proposé la notion de contexte partagé pour rendre compte du partage de la situation en cours. Cette notion a pour origine les travaux de Sperber et Wilson (1989) qui rejettent une vision de la communication comme un processus d'encodage-décodage pour affirmer le rôle de processus inférentiels qui «compensent», d'une part, le caractère toujours lacunaire de l'accès à la cognition ou aux pensées d'autrui, celles-ci ne pouvant se réduire aux «messages » émis, et d'autre part, l'impossibilité d'une connaissance mutuelle absolue entre les acteurs. Sperber et Wilson ont développé le concept « d'environnement cognitif mutuel » avec l'idée que c'est l'environnement transitoire et dynamique qui constitue ce qui est commun entre les acteurs à chaque instant plutôt qu'un savoir partagé, sachant que ce n'est pas l'environnement en tant que tel qui importe mais l'environnement en tant qu'environnement cognitif.

L'environnement cognitif d'un individu est un ensemble de faits qui lui sont manifestes, c'est-à-dire tous les faits qu'il peut percevoir ou inférer. Il inclut non seulement tous les faits de son environnement dont il a pris connaissance, mais aussi tous ceux dont il est capable de prendre connaissance. Les mêmes faits et hypothèses peuvent être manifestes dans les environnements cognitifs de deux individus. Dans ce cas, ces environnements cognitifs se recoupent, et leur intersection donne lieu à un environnement cognitif partagé entre les deux individus (Salembier \& Zouinar, 2004). Un environnement cognitif mutuel correspond à la partie de l'environnement cognitif partagé dans laquelle l'identité des individus qui ont accès aux différents faits et hypothèses est elle-même manifeste (Sperber \& Wilson, 1989). Dans un environnement cognitif mutuel, tout fait manifeste est dit mutuellement manifeste. Ainsi, Sperber et Wilson (1989) ont substitué à la notion de savoir mutuel une notion plus «faible " mais plus dynamique et empiriquement plus adéquate: «la manifesteté mutuelle». La notion de contexte partagé (Salembier \& Zouinar, 2004) opère une délimitation dans l'environnement cognitif mutuel (Figure 2). Le contexte partagé est défini comme l'ensemble des informations ou événements contextuels mutuellement manifestes pour un ensemble d'acteurs, à un instant $t$ dans une situation donnée, compte tenu de leurs capacités perceptuelles et 
cognitives, des tâches qu'ils doivent réaliser, et de leur activité en cours. Dans cette optique, le partage de la situation en cours est inféré et appréhendé sur la base du partage d'informations contextuelles.

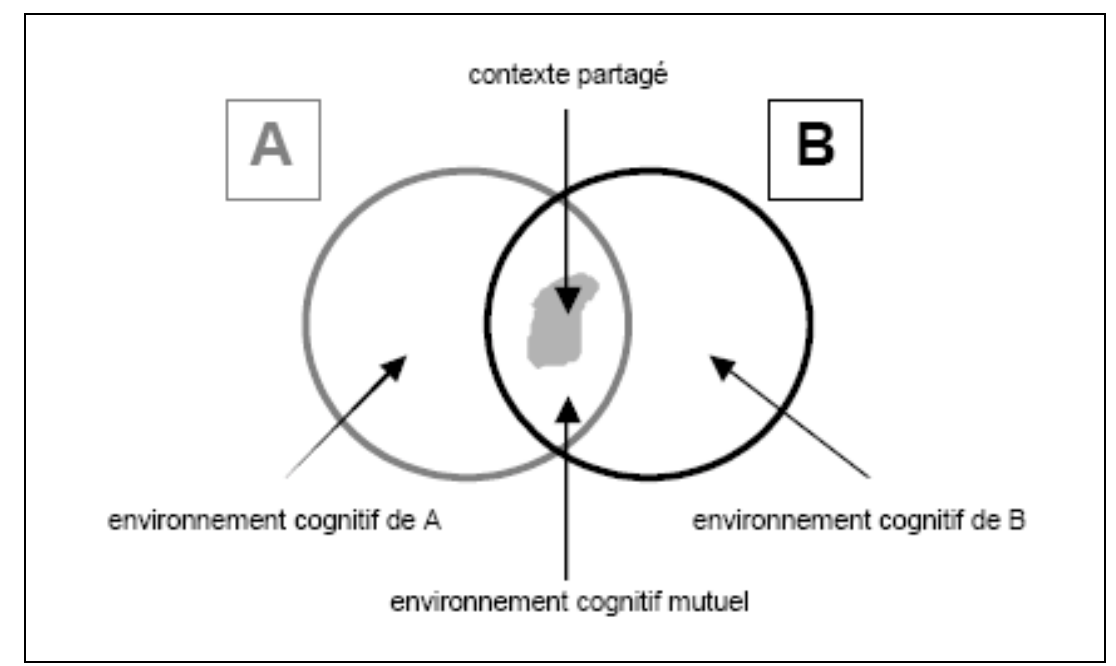

Figure 2 - Représentation graphique de la relation entre environnement cognitif mutuel et contexte partagé (Salembier et Zouinar, 2004).

\section{II.2.2.Le partage d'informations contextuelles en tennis de table}

Une étude menée en tennis de table a caractérisé les informations contextuelles partagées en articulant les propositions de Salembier et Zouinar (2004) et celles de Theureau (2006). Il s'agissait d'articuler les cours d'action de deux pongistes partenaires de double au cours d'un match afin d'identifier, à chaque instant du match, quelles étaient les informations contextuelles partagées par les deux joueurs (Poizat et al., 2008). Les résultats ont montré que ces informations concernaient essentiellement l'activité des autres joueurs (partenaires et adversaires). L'analyse a mis en évidence trois formes typiques de partage d'informations contextuelles entre les deux partenaires :

○ un contexte partagé symétrique. Dans ce cas les informations mutuellement manifestes pour les deux joueurs se référaient soit à un élément de la situation, soit à l'activité des deux pongistes (i.e., chacun des deux joueurs avait accès à des informations sur l'autre);

- un contexte partagé asymétrique. Dans ce cas les informations mutuellement manifestes pour les deux joueurs se référaient à l'activité d'un seul des deux pongistes. Les joueurs réalisaient des inférences identiques concernant l'un d'entre eux ; 
○ un non partage d'information. Dans ce cas aucune information n'était mutuellement manifeste pour les deux pongistes: ils ne réalisaient aucune inférence commune.

Ces trois types de contexte partagé alternaient au cours des matchs. Durant plusieurs points les pongistes ne partageaient aucune information contextuelle, au cours des points suivants il était symétrique, puis asymétrique, redevenait symétrique, etc. L'analyse n'a montré aucune régularité dans la succession de ces formes typiques de partage. Ce partage d'informations contextuelles était fluctuant et évoluait au cours du match en relation avec les interprétations réalisées par les joueurs. Lors des matchs étudiés, les moments de non partage d'information étaient plus nombreux que les moments de partage symétrique et asymétrique, sans que la performance ne soit altérée. Cette observation nous invite à penser que si la réalisation d'une performance collective par une dyade suppose un certain degré de partage d'informations contextuelles afin de s'assurer d'une compréhension mutuelle des actions de chacun, la coordination ne nécessite pas un partage d'informations contextuelles systématique.

\section{II.2.3. La vérification du partage d'informations contextuelles}

Un autre résultat majeur de cette étude en tennis de table est la mise en évidence de certaines activités destinées à vérifier le partage : afin d'apprécier la plausibilité des interprétations construites sur la base des informations contextuelles, les pongistes s'engageaient dans un processus de vérification du partage de ces informations. Deux processus de vérification ont été identifiés :

- vérifier le partage d'informations contextuelles de son point de vue. Ce processus consiste pour le joueur à s'assurer de la fiabilité des informations contextuelles sur lesquelles se basent ses propres interprétations ;

- vérifier le partage d'informations contextuelles du point de vue de son partenaire. Ce processus consiste pour le joueur à caractériser les informations contextuelles sur lesquelles pourraient se baser les interprétations de son partenaire.

Ces vérifications participent au degré de confiance, accordée par les pongistes, aux interprétations construites sur la base de ces informations contextuelles. Nous pouvons illustrer la manière dont les joueurs apprécient les interprétations susceptibles d'être effectuées par leur partenaire sur la base d'un exemple extrait d'un match de «double» disputé par une paire de pongistes constituée des joueurs nommés Jules et Paul. Lors de ce double, au score de 5-5 au cours du troisième set, Jules a enfreint une règle habituelle de fonctionnement de l'équipe. Dans cette équipe de double, le serveur informe ordinairement son partenaire du service qu'il va réaliser afin que celui-ci ne soit pas surpris par le retour de l'adversaire. Afin de masquer cette information aux adversaires, l'annonce du serveur à l'attention de son partenaire est réalisée, sous le plateau de la table, à l'aide de signes codifiés de la main. Au score de 5-5, Paul était serveur et a annoncé, en tendant l'index sous la table, qu'il allait réaliser un service coupé court. Jules s'est rapproché de Paul et a fait un mouvement circulaire de l'index sous la table pour lui demander de réaliser un « service latéral ». Jules a estimé que les services courts et coupés de Paul n'étaient plus assez performants à ce moment du match et qu'il fallait 
orienter le jeu de l'équipe vers l'offensive. Etant à ce moment, de son point de vue, le joueur le plus performant du double, Jules s'est permis d'enfreindre la règle de fonctionnement de l'équipe pour influencer l'activité de Paul. Face au caractère inhabituel de sa demande et aux exigences qu'elle imposait à son partenaire, Jules a estimé nécessaire de s'assurer de la compréhension de Paul. Il s'est placé de dos par rapport aux adversaires et a demandé oralement à son partenaire de réaliser un « service latéral ». Dans cet exemple, les éléments qui ont contribué à ce que Jules estime nécessaire de conforter le partage d'informations contextuelles par une communication verbale, sont la rupture d'une pratique habituelle concernant l'annonce du service (i.e., habituellement c'est le serveur qui choisit et annonce à son partenaire le service qu'il va réaliser) et la demande effectuée à Paul (i.e., réaliser un service non préférentiel et implicitement s'engager dans un jeu offensif). La communication verbale visait, pour Jules, à augmenter le degré et la pertinence des informations partagées, c'est-à-dire augmenter la visibilité de la requête et affirmer l'utilité de cette requête. Il cherchait à s'assurer de la compréhension de Paul afin de susciter chez lui un engagement identique au sien dans le point (i.e., «Etre offensif»). Dans cette situation, l'incertitude relative au partage d'informations contextuelles était jugée trop importante par Jules. Il a donc augmenté le degré de manifesteté des informations contextuelles par une communication verbale. Suite à cette communication, le degré de confiance qu'il accordait à ses propres inférences relatives aux interprétations de son partenaire a augmenté. De son point de vue, un partage d'information fiable a eu lieu entre les deux joueurs concernant le point à venir.

Ces processus de vérification soulignent que l'accès conjoint aux mêmes ressources de l'environnement et l'inter-visibilité ne garantissent pas le partage d'informations contextuelles. L'ensemble hétérogène de ressources disponibles que l'on nomme «contexte»n'est pas un réservoir dans lequel chacun irait puiser les mêmes informations (Decortis \& Pavard, 1994). Le partage d'informations contextuelles suppose une «activité compétente» des participants consistant à reconnaître les ressources informatives disponibles pour chacun (Robertson, 2002). Cette reconnaissance leur permet de juger de la fiabilité de leurs jugements et de ceux des autres protagonistes. Par exemple, en voile, les régatiers évaluent la fiabilité des jugements de leur partenaire en inférant les informations susceptibles d'être effectivement prises en compte par lui à chaque instant, compte tenu des contraintes spécifiques de sa tâche et de sa position dans le bateau (le barreur et l'équipier, du fait de leur position dans le bateau, n'ont pas la même visibilité du plan d'eau et n'ont pas accès aux mêmes informations). En déterminant quelles informations contextuelles sont effectivement accessibles ou non pour leur partenaire, ils apprécient la fiabilité des informations transmises par ce dernier (Saury, 2008b). Aussi les acteurs oscillent constamment entre doute et certitude concernant le partage d'informations contextuelles. Pour répondre à cette incertitude, ils déploient une activité cognitive spécifique pour juger de la fiabilité des interprétations construites sur la base de ces informations.

\section{II.2.4.La régulation du partage d'informations contextuelles}

Les résultats de cette première étude en tennis de table sur la vérification du partage d'informations contextuelles peuvent être complétés par d'autres 
résultats concernant la régulation de ce partage. La régulation du partage a été analysée dans deux types de dyades sportives : doubles de tennis de table, et équipages en voile (Poizat et al., 2008, 2009 ; Saury, 2008b). Les résultats ont mis en évidence le rôle de cette régulation dans la construction de l'intelligibilité mutuelle (Salembier \& Zouinar, 2004) entre les partenaires, autrement dit de la possibilité de comprendre les interprétations et les attentes de son partenaire. Le partage et l'intelligibilité mutuelle fluctuaient en permanence au cours des interactions du fait de cinq formes d'activités : enquête, mise en visibilité, surveillance, masquage, et recherche d'influence. Ces cinq formes d'activités se retrouvent dans ces deux contextes de performance (tennis de table et voile) mais s'actualisent de manière différente selon les contraintes des situations (pression temporelle, possibilités de communications verbales, possibilités d'accès des adversaires aux communications entre les partenaires, possibilités d'accès de chacun des partenaires aux informations contextuelles).

L'enquête vise à construire et évaluer le partage d'informations et l'intelligibilité mutuelle. Elle consiste à rechercher des informations pour inférer les interprétations et jugements de son partenaire et évaluer le degré de confiance pouvant être accordée à ceux-ci. En voile, lorsque l'un des deux régatiers s'aperçoit qu'il ne perçoit pas la situation de la même manière que son partenaire (par exemple, ce dernier demande de virer de bord alors qu'il estime quant à lui qu'il faut continuer sur le même bord), il s'engage dans une activité exploratoire afin d'obtenir des informations supplémentaires sur la situation. Ces informations l'amènent à augmenter ou diminuer le degré de fiabilité accordé aux interprétations de son partenaire. En tennis de table, cette enquête consiste à évaluer la pertinence des choix tactiques de son partenaire sur la base de l'efficacité des coups techniques réalisés.

La mise en visibilité consiste à informer son partenaire de ses interprétations, intentions et actions futures afin de faciliter et maintenir le partage d'informations et l'intelligibilité mutuelle. En tennis de table, cette mise en visibilité se réalise principalement à l'aide de comportements donnant à voir à son partenaire ses propres intentions. Par exemple, au moment du service, les joueurs adoptent des postures significatives afin d'informer leur partenaire du type de service qu'ils vont réaliser. La signification de ces postures doit être accessible au partenaire sans l'être aux adversaires. Elle se construit au cours des expériences communes passées. En voile, cette mise en visibilité s'effectue principalement par la continuité des communications verbales entre les deux partenaires au cours de la régate. Par ces communications, les régatiers informent l'autre des jugements qu'ils portent sur la situation.

La surveillance consiste à prendre régulièrement des informations sur la situation et son partenaire afin de s'assurer de l'intelligibilité mutuelle et du maintien d'un partage d'informations contextuelles. En voile, cette surveillance s'effectue notamment par le contrôle de la congruence entre les informations transmises par le partenaire et ses propres jugements de la situation. En tennis de table, elle s'effectue plus par des jeux de regards entre les partenaires.

Le masquage consiste à cacher à son partenaire certaines de ses interprétations. Un des deux protagonistes perçoit une divergence dans les interprétations et une diminution de l'intelligibilité mutuelle mais décide de ne 
pas en informer son partenaire, afin de préserver la viabilité du fonctionnement de la dyade. En tennis de table, ce masquage se produit, par exemple, lorsque l'un des deux joueurs doute de la fiabilité de son partenaire ou de la sienne : il tente de sécuriser son partenaire sans donner à voir ses propres inquiétudes. En voile, ce phénomène de masquage se produit lorsque l'un des régatiers, bien que ne partageant pas les interprétations de son partenaire, estime qu'une confrontation des points de vue serait nuisible à l'efficacité de la performance collective du fait des contraintes de la situation (pression temporelle par exemple).

La recherche d'influence consiste en une tentative de re-construction d'une intelligibilité mutuelle perçue comme déficiente. Ayant perçu une divergence entre ses propres interprétations et celles de son partenaire, le sportif tente d'influencer les interprétations et jugements de celui-ci. En voile, cette recherche d'influence s'exerce par le biais de la communication d'informations non congruentes avec celle qui sont communiquées par son partenaire afin d'orienter ses perceptions et interprétations. Pour augmenter l'efficacité de cette influence, les régatiers exagèrent quelquefois le caractère contradictoire de ces informations. En tennis de table, cette recherche d'influence se réalise par de brèves communications verbales et la réalisation de coups techniques qui vont orienter le décours du jeu.

\section{II.2.5. Intelligibilité mutuelle et performance collective}

Les résultats précédents confirment l'idée que l'intelligibilité mutuelle n'est jamais pré-donnée (Decortis \& Pavard, 1994). Elle se co-construit au cours de la situation en relation avec les interprétations précédemment réalisées, les informations contextuelles partagées et non partagées, le degré de confiance accordée à l'activité du partenaire, la sensibilité aux événements survenant dans la situation. Le contexte partagé et l'intelligibilité mutuelle présentent un caractère dynamique et incertain : ils résultent de processus inférentiels de la part des acteurs et sont en permanence négociés. Cette négociation s'accompagne de « luttes d'influence » entre les différents protagonistes, et une performance collective résulte de l'enchaînement «d'interactions coopératives » et «d'interactions non coopératives », voire « concurrentielles » (Saury, 2008a).

Lors des interactions coopératives, chacun des partenaires estime que l'activité de l'autre est congruente avec sa propre activité, et facilite la performance collective. Elles correspondent à des moments où chacun des membres de l'équipe perçoit l'activité du partenaire comme une « interférence positive » et/ou cherche à créer une "interférence positive» pour son partenaire (Castelfranchi, 1998; Hoc, 2003). Lors de ces moments, les partenaires construisent des interprétations convergentes, ont des attentes communes, partagent des informations contextuelles, présentent des ajustements mutuels basés sur la prise en considération de l'activité de l'autre comme aide pour sa propre activité.

Lors des interactions concurrentielles, un des partenaires estime que l'activité de l'autre est un frein à sa propre activité et à la performance collective. Elles correspondent à des moments où un des membres de l'équipe perçoit l'activité du partenaire comme une "interférence négative», et/ou 
cherche à créer une «interférence négative» pour son partenaire (Castelfranchi, 1998; Hoc, 2003). Lors de ces moments les partenaires construisent des interprétations divergentes, ont des attentes différentes, sont sensibles à des éléments différents de la situation. Cette divergence des points de vue peut conduire à une exploration de l'activité du partenaire, un masquage de ses propres interprétations, une surveillance accrue de la situation et/ou du partenaire, et une recherche d'influence des interprétations et de l'activité du partenaire.

Cette mise en évidence d'alternance d'interactions coopératives et concurrentielles entre des partenaires engagés dans la réalisation d'une tâche commune amène à repenser la notion de performance collective. La réalisation d'une performance collective n'exclut pas les conflits et les divergences de points de vue. Elle ne peut se réduire à une activité coopérative dans laquelle chacun cherche à faciliter l'activité de l'autre: elle intègre à la fois des processus coopératifs et concurrentiels (Saury, 2008b). Ces processus concurrentiels servent la performance collective en offrant des occasions de réguler le partage des informations contextuelles. Cette imbrication de processus coopératifs et compétitifs se retrouve dans d'autres situations, notamment les situations de travail. A titre d'illustration Gatewood (1984) a caractérisé, lors d'une étude sur la pêche au saumon en Alaska, la manière dont certains patrons de bateaux coordonnaient leurs actions et s'informaient mutuellement lors de leur recherche de bancs de poissons. Elle fait également écho à la notion de « coopétition », qui a été proposée par Nalebuff et Brandenburger (1996) pour désigner l'association de comportements stratégiques de coopération et de compétition simultanés de la part de deux ou plusieurs entreprises. Le fait que la simultanéité de ces comportements se retrouve entre des adversaires (dans le cas des entreprises mais aussi des sportifs) et des partenaires d'une même équipe nous invite à penser que coopération et compétition sont des facettes indissociables de la construction de toute activité collective (Saury, 2008b).

\section{II.3. Les modes de coordination et de partage au sein d'une équipe de sport collectif}

Si les études précédentes se sont attachées au fonctionnement de dyades sportives, récemment quelques études se sont intéressées à l'activité collective en sports collectifs et notamment en basket-ball (Bourbousson \& Sève, soumis ; Bourbousson et al., 2008).

\section{II.3.1. Les modes de coordination en basket-ball}

Une première étude s'est attachée aux modes de coordination entre les membres d'une équipe de joueurs de basket-ball (17-18 ans) de niveau National, sur la base de l'articulation des cours d'action des joueurs lors d'un match. L'étude des modes de coordination (Bourbousson et al., 2008) a été menée à partir de l'identification, pour chaque instant du match, des partenaires pris en compte pour agir par chacun des joueurs. L'analyse a mis en évidence quatre formats typiques d'organisation collective définis en relation avec les formes d'agencement des différentes coordinations locales et dyadiques au sein du réseau de joueur (Figure 3 ). Une «coordination locale » est un réseau de connexions mettant en relation deux joueurs ou plus, et une «coordination 
dyadique » un réseau de connexions mettant en relation exclusivement deux joueurs.

a)
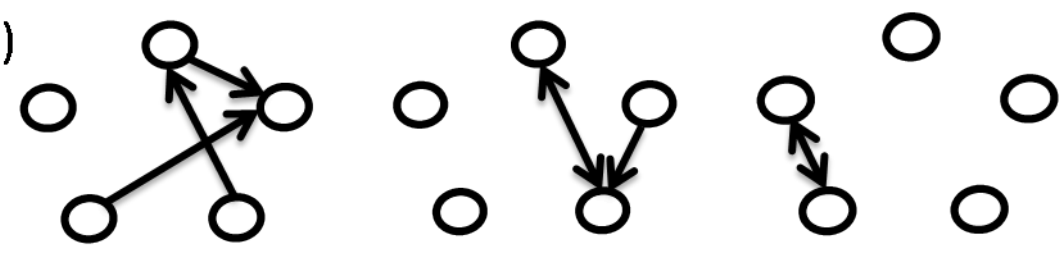

b)
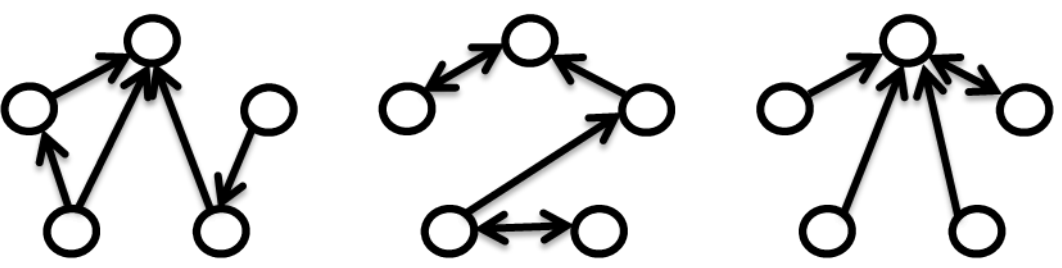

c)
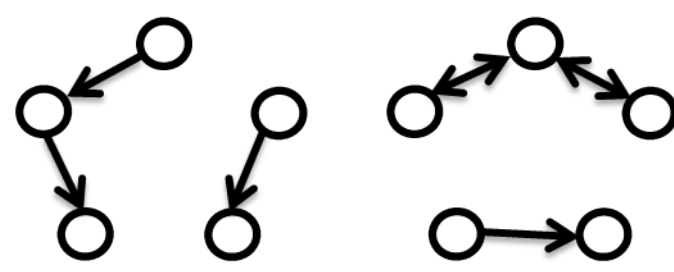

d)

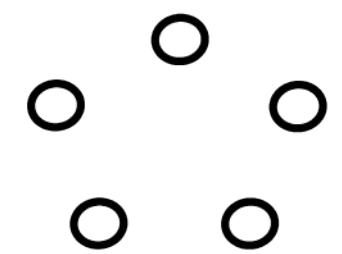

Figure 3 : Schématisation des formes typiques d'organisation collective : a) organisation collective par juxtaposition d'une coordination locale, avec une ou plusieurs activités individuelles,

b) organisation collective par imbrication en chaîne de coordinations dyadiques,

c) organisation collective par juxtaposition de deux coordinations locales,

d) organisation collective par juxtaposition des cinq activités individuelles. 
Ces quatre formes d'organisation collective s'enchaînaient dans le décours $\mathrm{du}$ match. Les deux premières constituaient les modalités de coordination les plus fréquentes (environ $90 \%$ du nombre d'organisations collectives observées). Par contre la quatrième était exceptionnelle ( $1 \%$ du nombre d'organisations collectives observées). Ceci pointe que le réseau cognitif de l'équipe se fondait essentiellement sur une imbrication de coordinations dyadiques. Les joueurs prenaient rarement en compte plus d'un partenaire pour agir, et les coordinations dyadiques transitoires constituaient un des ressorts majeurs du processus global de coordination de l'équipe. Du fait des coordinations locales entre les joueurs, l'équipe apparaît ainsi comme une entité dynamique qui se fait et se défait en permanence en relation avec les activités individuelles autonomes qui la constituent : des sous-réseaux se constituaient et disparaissaient, l'activité des joueurs les amenait à se déconnecter du reste de l'équipe de manière momentanée, des coordinations dyadiques s'imbriquaient.

$\mathrm{Au}$ sein de ce réseau d'interactions dynamique et transitoire, les joueurs participaient de manière différente à l'imbrication des coordinations dyadiques et à la coordination globale de l'équipe. En effet, des connexions cognitives étaient plus particulièrement privilégiées entre certains membres (du point de vue de leur fréquence d'occurrence dans le cours du match), de sorte que chaque joueur s'intégrait à un réseau de coordination spécifique. Les postes de jeu assignés par l'entraîneur aux joueurs au sein de l'équipe participent à la compréhension de la diversité de ces réseaux de coordination. Un poste de jeu précise, pour chaque joueur, les tâches spécifiques et le registre des actions qu'il doit privilégier. Par exemple, le «meneur de jeu» se voit attribuer la responsabilité d'annoncer (par une communication verbale ou un geste) à ses coéquipiers la routine de jeu à réaliser. Aussi lors des phases d'attaque, les coéquipiers sont souvent en attente du comportement du meneur pour savoir sur la base de quelle routine agir. Lors des phases de défense, le poste de meneur de jeu amène à être spatialement positionné en avant de ses coéquipiers : aussi il est facilement visible de ses partenaires. Ces éléments permettent de comprendre pourquoi lors du match étudié, le meneur a été le joueur le plus souvent pris en compte pour agir par chacun de ses partenaires, et a été le joueur le moins déconnecté du réseau cognitif global de coordination. Son activité servait souvent de support à la coordination de l'équipe, ses coéquipiers s'y référant fréquemment pour décider de leurs propres actions. A l'inverse, le joueur occupant le poste «d'intérieur» a été le joueur le moins souvent pris en compte par ses partenaires. Les caractéristiques de ce poste permettent également de comprendre cette faible prise en compte. Le joueur occupant le poste "d'intérieur » a pour tâche de jouer près du panier pour récupérer le ballon dans cette zone. Lors des phases d'attaque il est moins souvent en possession du ballon que ses partenaires, et lors des phases de défense, il est positionné derrière ses partenaires, se rendant ainsi moins visible. Ces résultats confirment que la répartition des rôles des membres de l'équipe influe sur les modalités de connexion cognitive entre les membres de l'équipe, et pointent le caractère dynamique et fluctuant des formes d'organisation collective et du « réseau cognitif de coordination » au cours de la réalisation d'une performance d'équipe. 


\section{II.3.2. Des formes diverses de partage au sein de l'équipe}

Les informations contextuelles partagées par les membres de cette équipe de basket-ball, ont été appréhendées à partir de la documentation et de la comparaison systématique, à chaque instant du match, des attentes, des perceptions et jugements sur la situation des différents joueurs. L'analyse a corroboré les modalités de coordination cognitive décrites précédemment, en montrant que le partage se réalisait à un niveau local, c'est-à-dire entre certains partenaires seulement. Au sein de l'équipe, le recoupement des attentes, des perceptions et jugements sur la situation était peu fréquent : la majorité des points de recouvrement se réalisaient à un niveau local (entre deux ou trois joueurs) et étaient ponctuels. A un même instant, deux ou trois joueurs pouvaient partager certains éléments et leurs partenaires d'autres éléments. Ces points de recouvrement se modifiaient constamment dans leur topologie et leur contenu, rendant la topologie de l'équipe relativement hétérogène du point de vue du partage. Aussi selon les moments du match, les formes de partage était très diverses. La Figure 4 présente différents formats de partage selon les modalités d'organisation collective de l'équipe, et le rôle central de certains joueurs dans cette organisation collective.

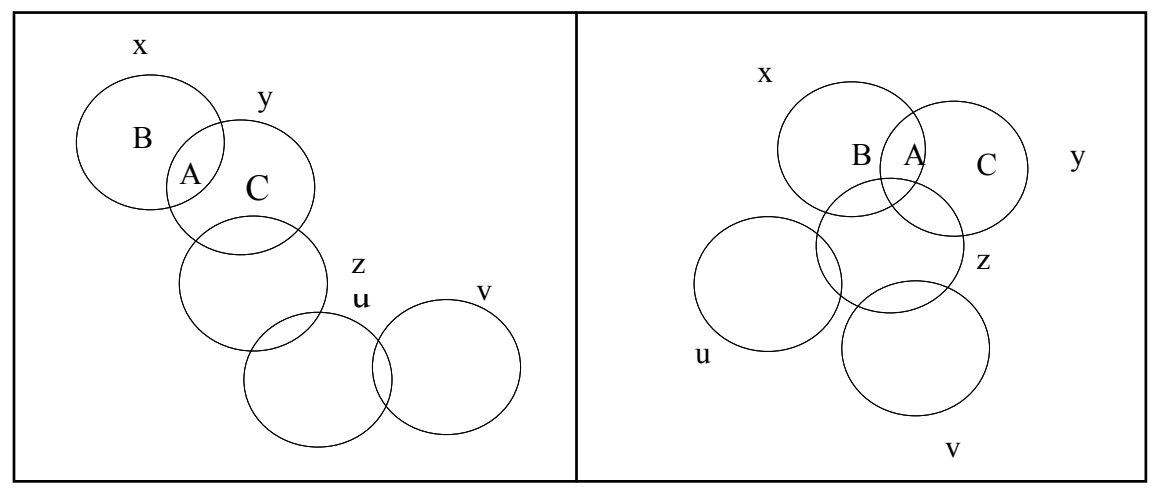

Figure 4 : Représentation graphique de deux cas illustratifs du partage dans une équipe de basket-ball.

Note : Par exemple, $\mathrm{x}, \mathrm{y}, \mathrm{z}, \mathrm{u}$ et $\mathrm{v}$ représentent des joueurs. $\mathrm{B}$ et $\mathrm{C}$ représentent les domaines de compréhension de $\mathrm{x}$ et $\mathrm{y}$. A représente une zone locale de partage entre $\mathrm{x}$ et $\mathrm{y}$.

Cette diversité des formes de partage observées (qui délimitent les formes d'organisation collective) nous invite à penser que le fonctionnement d'une équipe de basket-ball emprunte à la fois aux trois modalités de partage : similarité, complémentarité, et distribution (Cooke et al., 2007). Ces trois formes de partage alternent dynamiquement selon les moments du match. Le partage, en tant que similarité, caractérise la compréhension partagée localement entre certains partenaires, mais concerne rarement, du moins à un instant $\mathrm{t}$ du match, l'ensemble des joueurs simultanément. Une définition de l'organisation collective comme «constellation» d'îlots de compréhension locale permet à la fois de rendre compte d'un recoupement local des perspectives (partage entre certains joueurs), et d'une distribution de la compréhension des événements au sein de l'ensemble du collectif (plusieurs zones de partage au sein de l'équipe). 


\section{II.4. Apports principaux des études en sport}

$\mathrm{Au}$ total, ces résultats offrent de nouveaux éléments de compréhension concernant la cognition et la performance collectives d'équipes sportives mais aussi plus largement de toute équipe visant l'accomplissement d'une performance collective : équipe de travail, équipe d'intervention en milieu hostile (militaires, pompiers, sauveteurs), ensemble de création artistique, etc. Ils mettent en évidence en quoi l'intelligibilité mutuelle entre les membres de l'équipe constitue un des ressorts essentiels de la performance collective. Cette intelligibilité mutuelle est à la base d'une compréhension partagée de la situation présente, et facilite la coordination des activités individuelles en permettant aux membres de l'équipe de prévoir et anticiper les actions et besoins des autres membres. Elle permet la construction d'interprétations communes - ou congruentes - des événements, la formation d'attentes ou d'anticipations adéquates relatives aux actions des autres protagonistes, des prises de décisions congruentes par les différents membres de l'équipe.

L'intelligibilité mutuelle se fonde non seulement sur le partage de connaissances relativement stables et pré-existantes à l'activité collective mais aussi sur le partage de la situation présente (i.e., le partage d'informations contextuelles). Le partage d'informations contextuelles présente un caractère indéterminé et fluctuant. Ceci a trois conséquences essentielles :

- la mise en oeuvre d'activités spécifiques de la part des membres de l'équipe pour co-construire, vérifier et réguler le partage d'informations contextuelles. Le partage d'informations contextuelles suppose ainsi une « activité compétente » des participants consistant à reconnaître les ressources informatives disponibles pour chacun. Cette reconnaissance permet de construire et maintenir une intelligibilité mutuelle entre les différents partenaires de l'équipe, et d'évaluer la fiabilité des interprétations et jugements des différents protagonistes ;

- des modalités diverses de partage et de coordination. Si la réalisation d'une performance collective suppose un certain degré de partage d'informations contextuelles afin de s'assurer d'une compréhension mutuelle des actions de chacun, celle-ci ne nécessite pas un partage d'informations contextuelles systématique. Des points de raccordement « ponctuels » (Gatewood, 1984) sont suffisants pour une production collective coordonnée. Une performance collective émerge de l'interaction de compréhensions individuelles qui se raccordent localement et ponctuellement, sans qu'aucun individu, bien souvent, ne possède en propre une représentation et une compréhension de la totalité de la situation. Du fait de cette interaction de compréhensions individuelles se raccordant localement et ponctuellement, les modes de coordination sont fluctuants et diversifiés au sein de l'équipe ;

- l'alternance d'interactions coopératives et concurrentielles entre des partenaires engagés dans la réalisation d'une performance collective. Les processus concurrentiels servent la performance collective en offrant des occasions de réguler le partage des informations contextuelles, et de reconstruire une intelligibilité mutuelle perçue comme défaillante. 


\section{CONCLUSION}

Le chantier qui vise à développer des outils théoriques et méthodologiques pour accéder aux processus sous-jacents à la réalisation de performances collectives, tout en étant d'un intérêt majeur, est encore peu développé. Les premiers travaux en psychologie du travail ont appréhendé la performance collective en identifiant les fondements individuels de l'activité collective, sur la base de l'étude des connaissances communes et partagées. Cette perspective permet d'aborder les conditions de la performance collective mais sans appréhender certaines dimensions fondamentales de l'activité collective, notamment les processus de coordination. Aussi cette option théorique et méthodologique rend peu compte du domaine de phénomènes propre à la dynamique de l'activité collective émergeant des interactions entre acteurs, et se trouve limitée pour expliquer en quoi une «équipe experte» ne peut être confondue avec une « équipe d'experts ».

Pour rendre compte de la dynamique de l'activité collective, les auteurs explorent actuellement différentes voies en prenant le collectif comme unité d'analyse (e.g., Cooke et al., 2004 ; Huchins, 1995) ou en s'attachant à l'analyse de l'articulation des activités individuelles et de l'activité collective (e.g., Jeffroy et al., 2006). Il est essentiel que les chercheurs en psychologie du sport participent activement à ce renouveau des options théoriques et méthodologiques pour l'étude de l'activité et de la performance collectives. De par leurs caractéristiques (diversité des formats des équipes sportives, articulation d'objectifs antagonistes et agonistes, alternance de phases d'entraînement et de compétition, recherche d'excellence, affrontement délimité par un ensemble de règles formalisées dont le respect est assuré par un arbitre, importance des composantes sensori-motrices, affectives et cognitives de l'activité...), les situations de performance collective sportive forcent certains traits des situations d'interaction sociales. En mettant en exergue certains processus de la réalisation d'une performance collective, elles constituent des «situations d'étude privilégiées » pour l'analyse des processus inhérents à de telles performances ${ }^{1}$. Les quelques travaux menés dans le domaine du sport, en référence à ces nouveaux cadres d'analyse, ont permis de réinterroger les modélisations classiques de la performance collective en sport (Bourbousson et al., 2008; Poizat et al., 2008, 2009; Saury, 2008a), et pourraient également réinterroger d'autres types de collectifs et leurs modes de coordination. Audelà des enjeux théoriques, ces études ouvrent vers des perspectives pratiques nouvelles : comprendre la manière dont se co-construit la compréhension partagée entre des partenaires engagés dans une entreprise commune ouvre

\footnotetext{
${ }^{1}$ Par exemple, la revue Military Psychology a récemment publié un numéro spécial « Cognition, Competition, and Coordination: Understanding Expertise in Sports and its Relevance to Learning and Performance in the Military » (2008) qui souligne l'intérêt des recherches en psychologie du sport pour apporter des perspectives méthodologiques et théoriques nouvelles et pertinentes aux recherches en psychologie militaire.
} 
vers la conception de dispositifs visant à améliorer l'efficacité d'un collectif (e.g., Bourbousson \& Sève, sous presse).

\section{REFERENCES}

Bourbousson, J. \& Sève, C. (sous presse). Construction/déconstruction du référentiel commun d'une équipe de basketball au cours d'un match. Impulsions.

Bourbousson, J., Poizat, G., Saury, J., \& Sève, C. (2008). Caractérisation des modes de coordination interpersonnelle au sein d'une équipe de basket-ball. @ ctivité, 5, 2139.

Cannon-Bowers, J. A., \& Bowers, C. (2006). Applying work team results to sports teams: Opportunities and cautions. International Journal of Sport and Exercise Psychology, 4, 363-369.

Cannon-Bowers, J. A., Salas, E., \& Converse, S. A. (1993). Shared mental models in expert decision making teams. In N. J. Castellan (Ed.), Current issues in individual and group decision making (pp. 221-246). Hillsdale, NJ: Lawrence Erlbaum Associates.

Castelfranchi, C. (1998). Modelling social action for AI agents. Artificial Intelligence, $103,157-182$.

Cooke, N. J., \& Gorman, J. C. (2006). Assessment of team cognition. In P. Karwowski (Ed.), International encyclopedia of ergonomics and human factors (pp. 270-275). London: Taylor \& Francis Ltd.

Cooke, N. J., Gorman, J. C., \& Winner, J. (2007). Team cognition. In F. Durso, R. Nickerson, S. Dumais, S. Lewandowsky \& T. Perfect (Eds.), Handbook of applied cognition (pp. 239-268). New York: Wiley.

Cooke, N. J., Salas, E., Kiekel, P. A., \& Bell, B. (2004). Advances in measuring team cognition. In E. Salas \& S. M. Fiore (Eds.), Team cognition: Understanding the factors that drive process and performance (pp. 83-106). Washington, DC: APA.

Cooke, N. J., Stout, R. J., \& Salas, E. (2001). A knowledge elicitation approach to the measurement of team situation awareness. In M. McNeese, M. Endsley \& E. Salas (Eds.), New trends in cooperative activities: System dynamics in complex settings (pp. 114-139). Santa Monica, CA: Human Factors.

Decortis, F., \& Pavard, B. (1994). Communication et coopération : De la théorie des actes de langage à l'approche ethnométhodologique. In B. Pavard (Ed.), Systèmes coopératifs : De la modélisation à la conception (pp. 21-50). Toulouse : Octarès.

Eccles, D. W., \& Johnson, M. B. (2009). Letting the social and cognitive merge: New concepts for an understanding of group functioning in sport. In S. D. Mellalieu \& S. Hanton (Eds.), Applied sport psychology advances: A review (pp. 281-316). London: Routledge.

Eccles, D. W., \& Tenenbaum, G. (2004). Why an expert team is more than a team of experts: A social-cognitive conceptualization of team coordination and communication in sport. Journal of Sport and Exercise Psychology, 26, 542-560.

Eccles, D. W., \& Tenenbaum, G. (2007). A social cognitive perspective on team functioning in sport. In G. Tenenbaum \& R. C. Eklund (Eds.), Handbook of sport psychology (pp. 264-283). New York: Wiley.

Endsley, M. R. (1995). Toward a theory of situation awareness in dynamic systems. Human Factors, 37, 32-64.

Ericsson, K. A., \& Simon, H. A. (1980). Verbal reports as data. Psychological review, 
$87,215-251$.

Fiore, S. M., \& Salas, E. (Eds.) (2006). Team Cognition and expert teams: Emerging insights into learning and performance for exceptional teams [Special issue]. International Journal of Sports and Exercise Psychology, 4.

Gatewood, J. B. (1984). Cooperation, competition, and synergy: Information-sharing groups among southeast Alaskan salmon seiners. American Ethnologist, 11, 350370.

Gorman, J., Cooke, N., \& Winner, J. (2006). Measuring team situation awareness in decentralized command and control environments. Ergonomics, 49, 1312-1325.

Hazlehurst, B., McMullen, C. K., \& Gorman, P. N. (2007). Distributed cognition in the heart room: how situation awareness arises from coordinated communications during cardiac surgery. Journal of Biomedical Informatics, 40, 539-51.

Hinsz, V. B., Tindale, R. S., \& Vollrath, D. A. (1997). The emerging conceptualization of groups as information processors. Psychological Bulletin, 121, 43-64.

Hoc, J. M. (2001). Toward a cognitive approach to human-machine cooperation in dynamic situations. International Journal of Human-Computer Studies, 54, 509540.

Hoc, J. M. (2003). Coopération humaine et systèmes coopératifs. In G. Boy (Ed.), Ingénierie cognitive. IHM et cognition (pp. 139-187). Paris : Hermès.

Hutchins, E. (1995). Cognition in the wild. Cambridge, MA: MIT Press.

Jeffroy, Theureau, \& Haradji, (2006). Relation entre activité individuelle et activité collective. Confrontation de différentes démarches d'études. Toulouse : Octarès.

Lim, B. C., \& Klein, K. J. (2006). Team mental models and team performance: A field study of the effects of team mental model similarity and accuracy. Journal of Organizational Behavior, 27, 403-418.

Loiselet, A., \& Hoc, J.-M. (2001). La gestion des interférences et du référentiel commun dans la coopération : implications pour la conception. Psychologie Française, 46, 167-179.

Mathieu, J. E., Heffner, T. S., Goodwin, G. F., Salas, E., \& Cannon-Bowers, J. A. (2000). The influence of shared mental models on team process and performance. Journal of Applied Psychology, 85, 273-283.

Nalebuff, B., \& Brandenburger, A. (1996). La co-opétition, une révolution dans la manière de jouer concurrence et coopération. Paris : Village mondial.

Navarro, C. (1991). Une analyse cognitive de l'interaction dans les activités de travail. Le Travail Humain, 54, 113-128.

Pedersen, H. K., \& Cooke, N. J. (2006). From battle plans to football plays: Extending military team cognition to football. International Journal of Sport \& Exercise Psychology, 4, 422-446.

Poizat, G., Bourbousson, J., Saury, J., \& Sève, C. (2009). Analysis of contextual information sharing during table tennis matches: An empirical study on coordination in sports. International Journal of Sport \& Exercise Psychology. 7. 465-487.

Poizat, G., Sève, C., \& Saury, J. (2007, Septembre). Intelligibilité mutuelle et construction de signification dans les interactions homme-homme : un exemple en tennis de table. In P. Salembier \& A. Giboin (Chairs), L'intelligibilité mutuelle dans la coopération homme-homme et la coopération homme-machine. Communication présentée en session thématique du Congrès EPIQUE'07, Nantes.

Poizat, G., Sève, C., Serres, G., \& Saury, J. (2008). Analyse du partage d'informations contextuelles dans deux formes d'interaction sportives : coopérative et 
concurrentielle. Le Travail Humain, 71, 323-357.

Resnick, L. B., Levine, J. M., \& Teasley, S. D. (Eds.) (1991). Perspectives on socially shared cognition. Washington, DC: APA.

Robertson, T. (2002). The public availability of actions and artefacts. Computer Supported Cooperative Work, 11, 299-316.

Salas, E., \& Fiore, S. M. (Eds.) (2004). Team cognition: Understanding the factors that drive process and performance. Washington, DC: APA.

Salas, E., Dickinson, T. L., Converse, S. A., \& Tannenbaum, S. I. (1992). Toward an understanding of team performance and training. In In R. W. Swezey \& E. Salas (Eds.), Teams: Their training and performance (pp. 3-29). Norwood, NJ: Ablex.

Salas, E., Prince, C., Baker, D. P., \& Shrestha, L. B. (1995). Situation awareness in team performance: Implications for measurement and training. Human Factors, 37, 123-136.

Salembier, P., \& Zouinar, M. (2004). Intelligibilité mutuelle et contexte partagé : inspirations théoriques et réductions technologiques. @ctivités, 1,64-85.

Salmon, P., Stanton, N., Walker, G., \& Green, D. (2006). Situation awareness measurement: A review of applicability for C4i environments. Applied Ergonomics, $37,225-238$.

Sarter, N. B., \& Woods, D. D. (1991). Situation awareness: A critical but ill-defined phenomenon. International Journal of Aviation Psychology, 1, 45-57.

Saury, J. (2008a). La coopération dans les situations d'intervention, de performance et d'apprentissage en contexte sportif. Contribution au développement d'un programme de recherche en ergonomie cognitive des situations sportives en STAPS. Note de synthèse d'Habilitation à Diriger des Recherches, Université de Nantes.

Saury, J. (2008b). Transitions entre formes coopératives et concurrentielles de l'activité collective dans la prise de décision tactique au sein d'équipages experts en voile. In J.M. Hoc \& Y. Corson (Eds.), Actes du Congrès 2007 de la Société Française de Psychologie (pp. 177-185).

Sperber, D., \& Wilson, D. (1989). La pertinence. Communication et cognition. Paris : Les Editions de Minuit.

Stout, J., Cannon-Bowers, J.A., \& Salas, E. (1996). The role of shared mental models in developing team situational awareness: Implications for training. Training Research Journal, 2, 85-116.

Stout, R., Cannon-Bowers, J. A., Salas, E., \& Milanovich, D. M. (1999). Planning, shared mental models, and coordinated performance: An empirical link is established. Human Factors, 41, 61-71.

De Terssac, G., \& Chabaud, C. (1990). Référentiel opératif commun et fiabilité. In J. Leplat \& G. de Terssac (Eds.), Les facteurs humains de la fiabilité dans les systèmes complexes (pp. 111-139). Toulouse: Octarès.

Theureau, J. (2004). Le cours d'action : méthode élémentaire. Toulouse : Octarès.

Theureau, J. (2006). Cours d'action : méthode développée. Toulouse : Octarès.

Ward, P., \& Eccles, D. W. (2006). A commentary on "Team cognition and expert teams: Emerging insights into learning and performance for exceptional teams". International Journal of Sport and Exercise Psychology, 4, 463-483.

Wittenbaum, G. M., Stasser, G., \& Merry, C. J. (1996). Tacit coordination in anticipation of small group task completion. Journal of Experimental Social 
Psychology, 32, 129-152. 